\title{
Precision exploration of neutron spin structure at Jefferson Lab
}

\author{
Nilanga Liyanage \\ University of Virginia ,Charlottesville, Virginia
}

\begin{abstract}
Spin structure functions provide basic information about the spin of the quark distributions inside the nucleon. Experimental understanding of the nucleon spin in the kinematic region where the three basic ("valence") quarks dominate the nucleon wave function is still rather poor. Jefferson lab, with its high quality, high polarization continuous electron beam, and a high density polarized ${ }^{3} \mathrm{He}$ target in experimental Hall A, provides the ideal opportunity to gather neutron spin structure data in the valence region with unprecedented precision. Two high precision neutron spin structure measurements were completed in Hall A last summer. The first experiment measured the spin asymmetry $A_{n}^{1}$ in the valence region while in second experiment higher-twist effects were studied via measurements of $g_{2}^{n}$. The planed upgrade of Jefferson lab CEBAF accelerator to $12 \mathrm{GeV}$ will significantly increase the accessible kinematic range and the precision of these measurements.
\end{abstract}

\section{INTRODUCTION}

In the past three decades lepton-nucleon deep-inelastic scattering (DIS) experiments have provided us with extensive data on the sub-structure of the nucleon. A global analysis of the DIS data has not only established the quark-parton picture of the nucleon, but also extracted the distributions of the partons (valence quarks, sea quarks and gluons). In lepton-nucleon deep-inelastic scattering, the lepton interacts with the nucleon by exchanging a virtual photon with one of the quarks in the nucleon. This process is characterized by two variables, $Q^{2}$ and $x$. The quantity $Q^{2}$ is the four-momentum squared for the exchanged virtual photon, and $x=Q^{2} /(2 M v)^{1}$ is the fraction of the nucleon momentum carried by the struck quark (at the Bjorken scaling limit). The $Q^{2}$ evolution of the DIS data has helped establish Quantum Chromo dynamics (QCD) as the fundamental theory governing the strong interaction.

One of the most fundamental properties of the nucleon is the structure of its valence quark distributions. At small values of the scaling variable $x$, sea quarks (quarkantiquark pairs), shadow the effects of valence quarks. As $x$ increases beyond $\sim 0.3$, the sea quark contributions to the nucleon wave function becomes increasingly small. This allows for a direct, clean probe of the valence quark distributions at large values of $x(x>0.4)$ [1]. Unfortunately due to limitations at high energy laboratories, the quality of valence region data in many experimental

\footnotetext{
${ }^{1} M$ is the mass of the nucleon and $v$ is the energy transfer.
}

fronts is rather poor. The lack of precision experimental data in the valence region is most apparent in the case of spin observables for the neutrons. This is mainly due to technical difficulties with polarized beams and targets, low luminosities available at high energy facilities and lack of a free neutron target.

In the ground state of the ${ }^{3} \mathrm{He}$ nucleus the two protons pair-up in a S-state with their spins anti-aligned. As a result, the neutron in ${ }^{3} \mathrm{He}$ carries almost $90 \%$ of the nuclear spin making polarized ${ }^{3} \mathrm{He}$ an ideal source for polarized neutrons [2]. The high-luminosity polarized ${ }^{3} \mathrm{He}$ target in experimental Hall A at Jefferson Lab, combined with the Jefferson Lab high polarization electron beam, provides the best opportunity to gather precision spin observable data for the neutron in the valence region.

The cross section for inclusive scattering of polarized leptons off a polarized nucleon target depends on two unpolarized structure functions $F_{1}\left(x, Q^{2}\right)$ and $F_{2}\left(x, Q^{2}\right)$, and two polarized structure functions $g_{1}\left(x, Q^{2}\right)$ and $g_{2}\left(x, Q^{2}\right)$. In the Quark-Parton model $F_{1}$ and $F_{2}$ are directly related to quark distributions inside the nucleon while $g_{1}$ is determined by the quark spin distributions. Another important observable $A_{1}$, virtual photon asymmetry, is defined in terms of total photo-absorption cross sections $\sigma^{1 / 2}$ and $\sigma^{3 / 2}$

$$
A_{1}=\frac{\sigma^{1 / 2}-\sigma^{3 / 2}}{\sigma^{1 / 2}+\sigma^{3 / 2}}
$$

where super-scripts $1 / 2$ and $3 / 2$ indicate the total helicity of the photon-nucleon system.

In this paper we give a brief overview of two precision neutron spin structure measurements carried out in 


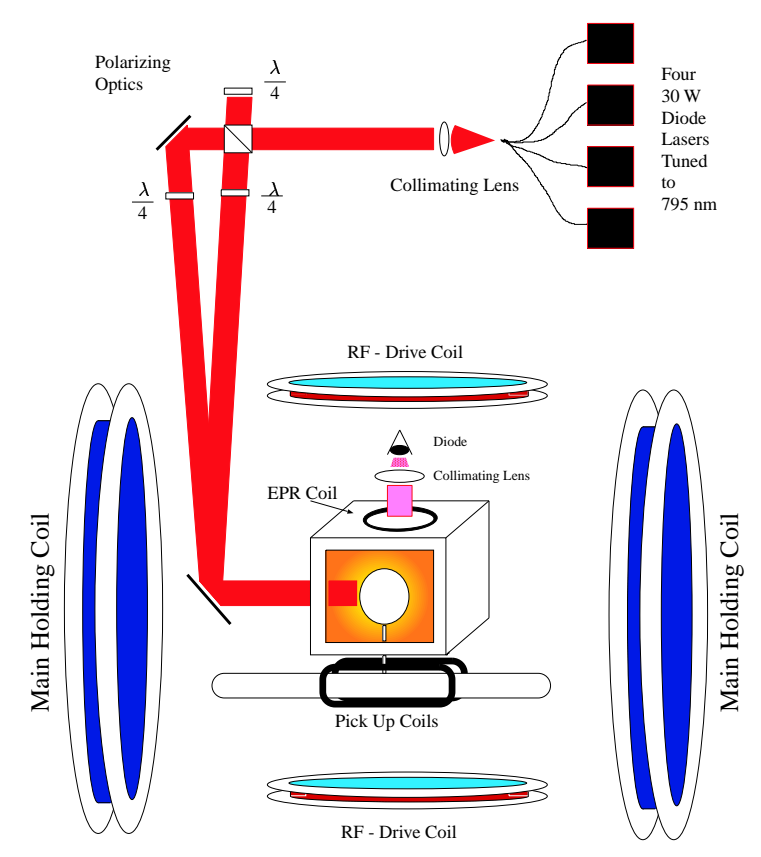

FIGURE 1. A schematic diagram of the hall A polarized ${ }^{3} \mathrm{He}$ target

experimental hall-A last Summer. Jefferson lab experiment 99-117 [3] used the hall A polarized ${ }^{3} \mathrm{He}$ target to measure virtual photon asymmetry for the neutron, $A_{1}^{n}$, with unprecedented precision up to $x \sim 0.61$. Experiment 97-103 [4] measured the $g_{2}$ structure function for the neutron. We will also describe some of the neutron spin physics opportunities that will become possible with the $12 \mathrm{GeV}$ beam at Jefferson Lab. Jefferson Lab $12 \mathrm{GeV}$ beam creates many other exciting opportunities to study the nucleon spin structure in inclusive, semi-inclusive and exclusive reactions in halls A, B and C. A detailed discussion of the proposed Jefferson Lab $12 \mathrm{GeV}$ physics program can be found in the Jefferson Lab $12 \mathrm{GeV}$ White-Paper [5].

\section{EXPERIMENTAL SETUP}

The Thomas Jefferson National Accelerator Facility, the world's premier medium energy electron scattering laboratory, is located in Newport News, Virginia. The facility consists of a state-of-the art continuous wave electron accelerator (CEBAF), three complementary experiment halls that utilize the beam to explore different aspects of nuclear physics, a free electron laser facility and an applied research center. CEBAF accelerator at Jefferson $\mathrm{Lab}$ is capable of simultaneously delivering high quality polarized electron beams of up to $6 \mathrm{GeV}$ to the three experiment halls. An upgrade of the accelerator and experimental equipment up to $12 \mathrm{GeV}$ is highlighted as a high priority in the Nuclear Science Advisory Committee (NSAC) Long Range Plan and is expected in the near future.

The experimental hall A is instrumented with an identical High Resolution Spectrometer (HRS) pair capable of detecting and momentum analyzing charged particles up to $4 \mathrm{GeV}$. In the experimental program described here, both spectrometers were used to detect electrons. To take the full use of the $12 \mathrm{GeV}$ beam, a new spectrometer, MAD (Medium Acceptance Device), is proposed for Hall A. This spectrometer will have a relatively large solid angle acceptance of $30 \mathrm{msr}$ while providing low background conditions so that the full available beamtarget luminosity can be used.

The heart of the Jefferson Lab Hall A neutron spin physics program is the polarized ${ }^{3} \mathrm{He}$ target constructed by the Jefferson Lab polarized ${ }^{3} \mathrm{He}$ collaboration. This polarized ${ }^{3} \mathrm{He}$ target is based on the principal of spin exchange between optically pumped alkali-metal vapor and noble-gas nuclei [11]. The main feature of the target is the sealed glass cell, which under operating conditions contains ${ }^{3} \mathrm{He}$ at about 10 atmospheres. As shown in Figure 1 the cell consists of two chambers; an upper chamber where the spin exchange takes place and a lower chamber through which the electron beam passes. The appropriate number density of the alkali-metal Rubidium in the upper chamber is maintained by keeping it at a temperature of $200^{\circ} \mathrm{C}$.

The main coils shown in Figure 1 are large Helmholtz coils used to apply a static magnetic field of about 25 Gauss. Also shown are the components for the NMR and EPR polarimetry. The optics system includes three diode lasers for longitudinal pumping and three for transverse pumping. A polarizing beam splitter lens system and quarter wave plates are required to condition each laser beam line and provide circular polarization.

An average target polarization of about $40 \%$ was achieved for the Summer-long run of two neutron spin experiments last year (E99-117 and E97-103).

\section{JEFFERSON LAB EXPERIMENT 99-117: PRECISION MEASUREMENTS OF THE SPIN ASYMMETRY $A_{1}^{N}$ IN THE HIGH-X REGION}

Virtual photon asymmetry is an important observable in the study of dynamics of spin-flavor symmetry breaking. Early quark-parton models of the nucleon that preserve exact $\mathrm{SU}(6)$ symmetry require that $A_{1}^{n}=0$ and $A_{1}^{p}=5 / 9$. However, in nature the spin-flavor $\mathrm{SU}(6)$ symmetry is broken. Modern quark models with broken SU(6) sym- 


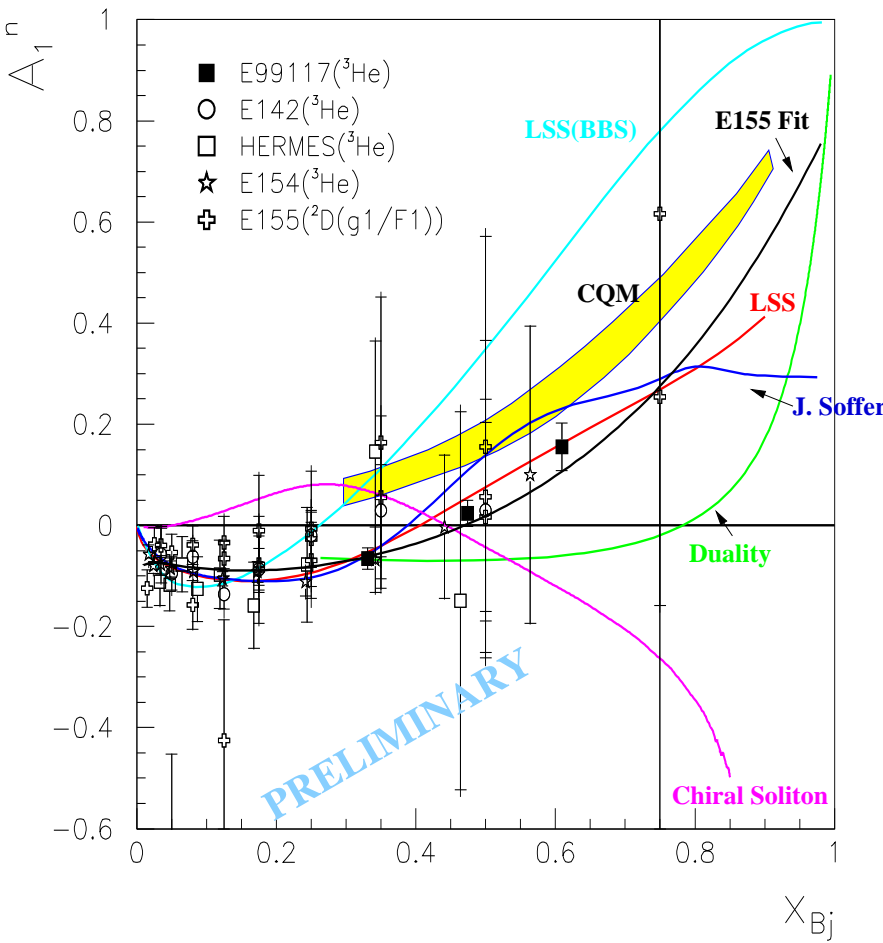

FIGURE 2. Preliminary result of $A_{1}^{n}$ from Jefferson Lab Experiment 99-117, compared with world data and theoretical predictions. Curves: predictions of $\frac{g_{1}}{F_{1}} \approx A_{1}$ from the pQCD NLO fit (LSS) to polarized DIS data [13], $A_{1}^{n}$ from constituent quark model (light shaded band) [1], pQCD based model using BBS parameterization (LSS(BBS)) [10, 12], statistical model at $\mathrm{Q}^{2}=4$ $(\mathrm{GeV} / \mathrm{c})^{2}$ (J. Soffer) [14], local duality based model [15], chiral soliton model at $\mathrm{Q}^{2}=3(\mathrm{GeV} / \mathrm{c})^{2}[16]$, and E155 fit at $\mathrm{Q}^{2}=4$ $(\mathrm{GeV} / \mathrm{c})^{2}$.

metry predict that both $A_{1}^{p} \rightarrow 1$ and $A_{1}^{n} \rightarrow 1$ as $x \rightarrow$ $1[1,6,7]$. Perturbative-QCD calculations [8, 9] at the $x \rightarrow 1$ limit also show that $A_{1}^{p} \rightarrow 1$ and $A_{1}^{n} \rightarrow 1$ as $x \rightarrow 1$. Given the fundamental information that can be extracted from the spin observables at high $x$, it is unfortunate that there has been virtually no experimental data for $A_{1}^{n}$ for $x \geq 0.4$. While firm pQCD predictions exist for $A_{1}^{n}$ as $x \rightarrow 1$ and $Q^{2} \rightarrow \infty$, the existing measurements at high $x$ lack the precision to even distinguish any of the predictions from the naive $\mathrm{SU}(6)$ result.

Jefferson lab experiment 99-117 measured $A_{1}^{n}$ over the $x$ range from 0.33 to 0.61 by measuring asymmetries for scattering of highly polarized $(80 \%) 5.7 \mathrm{GeV}$ electrons off hall A polarized ${ }^{3} \mathrm{He}$ target. All the data was within the Deep Inelastic Scattering region with $2.74<Q^{2}<$ $4.9(\mathrm{GeV} / \mathrm{c})^{2}$ and $4<W^{2}<6.5(\mathrm{GeV} / \mathrm{c})^{2}$. Beam current was limited to $15 \mu \mathrm{A}$ to minimize target depolarization. A double-arm Moller polarimeter was used to measure the beam polarization once every few days, a Compton polarimeter was used to monitor the beam polarization continuously.

The Hall-A High Resolution Spectrometer pair was used in a symmetric configuration to detect scattered electrons at two scattering angles and three momen- tum settings. At each kinematic point, asymmetries were measured with the target polarization aligned both parallel and perpendicular to the beam. To minimize systematic errors, data were taken for all four beam-target polarization direction configurations in the parallel case and in two configurations in the perpendicular case.

Analysis of the data from the experiment is almost complete. The nuclear corrections were applied using most recent models [17] to extract $A_{1}^{n}$ from $A_{1}^{3} \mathrm{He}$. As the preliminary results are in Fig. 2 indicates, E99-117 has greatly improved the precision of data in the high-x region and for the first time, a cross over of $A_{1}^{n}$ from negative to positive is observed. The availability of $12 \mathrm{GeV}$ beam at Jefferson Lab will allow the $A_{1}^{n}$ measurement to be extended to $x \sim 0.8$ with high precision. The anticipated data are shown in Fig. 3. While an invariant mass cut of $W>2 \mathrm{GeV}$, would allow the deep inelastic continuum to be cleanly accessed, one may extend the measurements of $A_{1}^{n}$ to even larger $x$ by using quark-hadron duality in the resonance region. 


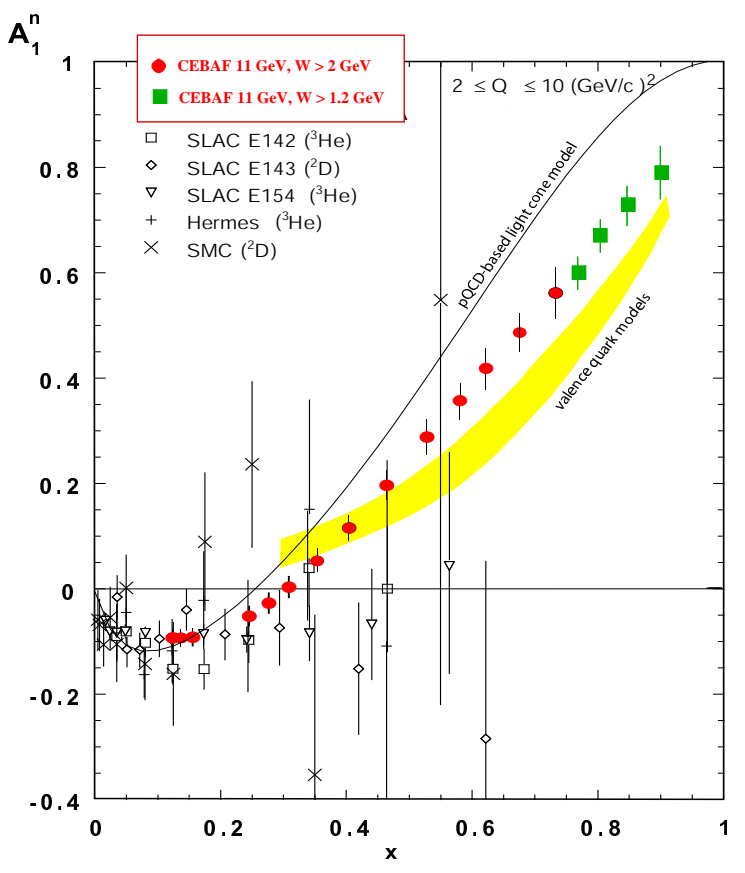

FIGURE 3. Projected data for a measurement of $A_{1}^{n}$ with 12 $\mathrm{GeV}$ beam at Jefferson Lab, The red filled circles are for the data in the DIS region $(W>2 \mathrm{GeV})$, while the filled squares show the possibility of extending the measurement to higher $x$ by relaxing the invariant mass cut.

\section{JEFFERSON LAB EXPERIMENT 97-103: SEARCH FOR HIGHER-TWIST EFFECTS IN THE NEUTRON SPIN STRUCTURE FUNCTION $G_{2}^{N}\left(X, Q^{2}\right)$.}

While the $g_{1}$ structure function has a simple interpretation in the quark-parton model in terms of quark helicity distributions and has been the focus of extensive experimental programs over the last decade, there have been few dedicated experimental studies of the $g_{2}$ structure function. The $g_{2}$ structure function is related to the transverse polarization of the nucleon, and although it does not have a simple quark-parton model interpretation, it contains important information about quark-gluon correlations within the nucleon. In QCD the quark-gluon correlations are associated with so-called higher twist operators (where "twist" is defined as the difference between dimension and spin of an operator), which are suppressed by additional factors of $1 / Q$ relative to the leading twist contribution (which is associated with free quark scattering). At large values of $Q^{2}, \mathrm{QCD}$ allows one to relate moments of spin structure functions to the matrix elements of operators of given twist. The simplest twist- 3 matrix element that contains information on quark-gluon correlations is given by:

$$
d_{2}\left(Q^{2}\right)=\int_{0}^{1} d x x^{2}\left[2 g_{1}\left(x, Q^{2}\right)+3 g_{2}\left(x, Q^{2}\right)\right]
$$

Note that because of the $x^{2}$ weighting in Eq.(2), $d_{2}$ is dominated by the large- $x$ behavior of $g_{1}$ and $g_{2}$. The physical significance of $d_{2}$ is that it reflects the response of a quark to the polarization of the gluon color field in the nucleon, $d_{2}=\left(2 \chi_{B}+\chi_{E}\right) / 3$, with $\chi_{B}\left(\chi_{E}\right)$ the gluon-field polarizability in response to a color magnetic (electric) field $\vec{B}(\vec{E})$.

Jefferson Lab experiment 97-103 made a precision measurement of $g_{2}^{n}$ at $x \approx 0.2$ for five different $Q^{2}$ values from 0.58 to $1.36 \mathrm{GeV}^{2}$. This experiment used the same setup as E99-117, except the target cell was $40 \mathrm{~cm}$ long. The main contribution to $g_{2}$ comes from the transverse asymmetry. Hence most of the data were taken with the target polarized perpendicular to the beam direction. Special attention was paid to the precise determination of the target polarization direction and to the control of beam helicity charge asymmetry.

The precision reached in E97-103 is an order of magnitude improvement over the best available world data. The data analysis is underway and final results are expected soon. These results will provide the first precision determination of higher-twist effects in $g_{2}^{n}$ above the leading twist component of $g_{2}^{n(W W)}$.

Availability of the $12 \mathrm{GeV}$ beam at Jefferson Lab will allow for a mapping of $g_{2}^{n}$ over $x$ and $Q^{2}$ with special focus on the high $x$ region which dominates $d_{2}$. These $g_{2}^{n}$ data will enable the extraction of the $d_{2}$ matrix element for the neutron with a factor of 10 statistical improvement in the error over the best available world data. Projected uncertainties for such an experiment are indicated by the squares in Fig. 4 .

\section{SUMMARY}

With the highest polarized luminosity in the world and flexibility in target polarization directions, the Jefferson Lab Hall-A polarized target provides a unique opportunity to gather high-precision neutron spin structure function data over a large kinematic range. Jefferson lab Experiment 99-117 provides the first high-precision $A_{1}^{n}$ data in the high-x region while Experiment 97-103 allows for the first precision study of higher-twist effects in neutron spin structure functions through a precise measurement of $g_{2}^{n}$. The proposed Jefferson lab upgrade to $12 \mathrm{GeV}$ will significantly increase the accessible kinematic range and the precision of these measurements. 


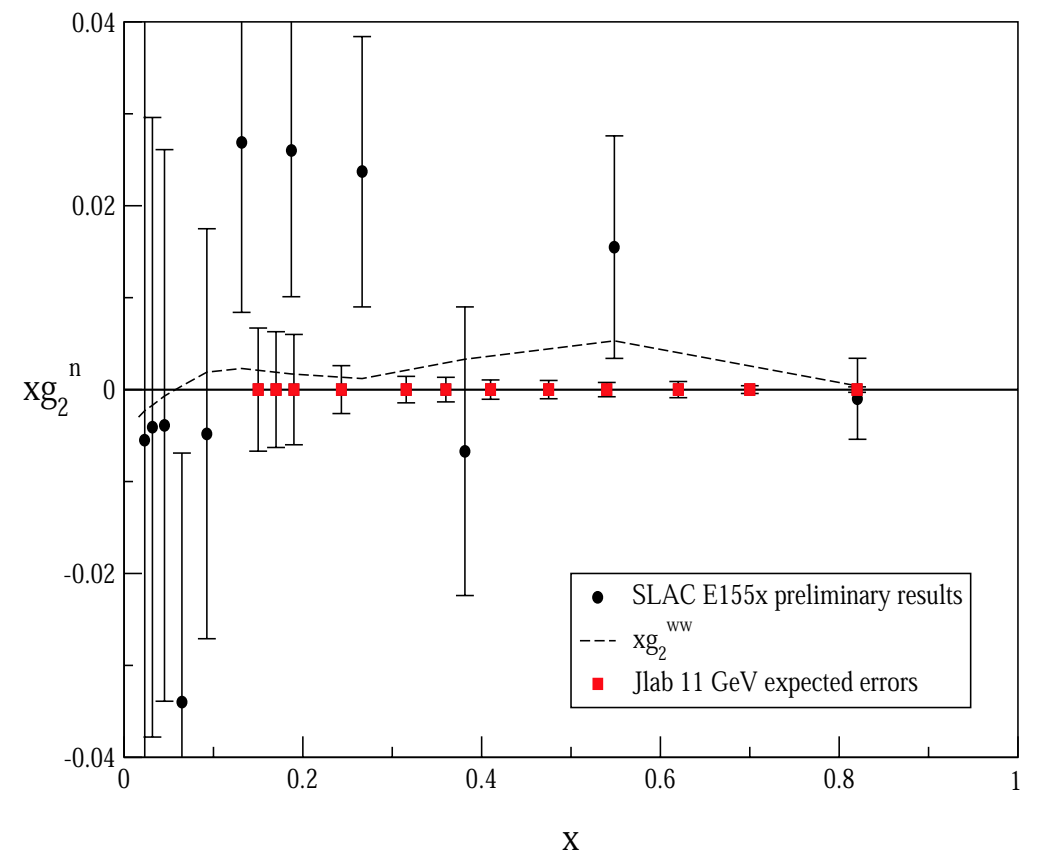

FIGURE 4. The preliminary results for the $g_{2}^{n}$ spin structure function from SLAC experiment E155x. The dashed curve shows the Wandzura-Wilczek [18] calculation of the leading twist contribution to $g_{2}^{n}$. The open squares are the expected uncertainties from an 11-GeV JLab measurement.

\section{ACKNOWLEDGMENTS}

The work presented here was supported in part by the U.S. Department of Energy (DOE). The Southeastern Universities Research Association operates Jefferson Lab for DOE under contract DE-AC05-84ER40150.

\section{REFERENCES}

1. N. Isgur, Phys. Rev. D 59, 034013 (1999).

2. J. L Friar et al., Phys. Rev. C 42, 2310 (1990).

3. Jefferson Lab experiment 99-117, Z. E. Meziani, J. P. Chen, P. Souder spokespersons.

4. Jefferson Lab experiment 97-103, Todd Averett and Wolfgang Korsch spokespersons.

5. The Science Driving the $12 \mathrm{GeV}$ Upgrade of CEBAF, Available at http://www.jlab.org/div_dept/physics_division/GeV/ WhitePaper_V11.pdf

6. F. Close, Phys. Lett. B 43, 422 (1973), Nucl. Phys. B 80, 269 (1974); and An Introduction to Quarks and Partons, Academic Press, N.Y., p. 197 (1976)

7. R. Carlitz, Phys. Lett. B58, 345 (1975)

8. S. J. Brodsky and G. R. Farrar, Phys. Rev. Lett. 31, 1153 (1973); Phys. Rev. D 11, 1309 (1975).

9. G. R. Farrar and D. R. Jackson, Phys. Rev. Lett. 35, 1416 (1975).

10. S. Brodsky, M. Burkhardt and I Schmidt, Nucl. Phys. B 441197 (1995)

11. N. R. Newbury, A. S. Barton, P. Bogoard, G. D. Cates, M.
Gatzke, H. Mabuchi and B. Saam, Phys. Rev. A 48, 558 (1993).

12. E. Leader, A. V. Sidorov, and D. B. Stamenov, Int. J. Mod. Phys. A 135573 (1998).

13. E. Leader, A. V. Sidorov, D. B. Stamenov, Eur. Phys. J. C23, 479 (2002).

14. C. Bourrely, J. Soffer, F. Buccella, Eur. Phys. J. C23, 487 (2002).

15. W. Melnitchouk, Phys. Rev. Lett. 86, 35 (2001). e-Print: hep-ph/0106073

16. H. Weigel, L. Gamberg and H. Reinhardt, Phys. Lett. B399, 287 (1997); Phys. Rev. D55, 6910 (1997). O. Schr oder, H. Reinhardt and H. Weigel; e-Print: hepph.9902322

17. C. Ciofi delgi Atti, S. Scopetta, E. Pace, and G. Salme, Phys. Rev. C 48, R969 (1993); C. Ciofi delgi Atti, and S. Scopetta, Phys. Lett. B404, 223 (1997).

18. S. Wandzura and F. Wilczek, Phys. Lett. B72, 195 (1977). 